\title{
Immunogenicity is predictive of future treatment success in RA
}

Patients with rheumatoid arthritis (RA) who fail to respond to an initial tumor necrosis factor (TNF) inhibitor are often prescribed an alternative anti-TNF agent. Jamnitski and colleagues report in Annals of the Rheumatic Diseases that the specific reason for the failure of the first therapy has implications for the success of the subsequent TNF inhibitor. "We hypothesized that nonresponse to a drug in the presence or absence of anti-drug antibodies will have consequences for the response to a second anti-TNF treatment," explains Gerrit Jan Wolbink, the study's lead investigator.

The investigators recruited 292 consecutive patients with RA who had received a new prescription for etanercept

(50 mg weekly or $25 \mathrm{mg}$ twice per week). Of these patients, 89 individuals-referred to as 'switchers'-had previously been treated with either infliximab $(n=30)$ or adalimumab ( $n=59)$; the remaining 203 patients were anti-TNF-naive.

The researchers measured the expression of anti-infliximab and anti-adalimumab antibodies in the switchers and found antibodies towards one of these drugs in approximately half of these patients. Disease activity was assessed in the three patient cohorts - anti-TNF-naive patients, switchers with anti-drug antibodies and switchers without anti-drug antibodies - at baseline and after 4, 16 and 28 weeks of etanercept therapy. Both 28-joint Disease Activity Score (DAS28) and European League Against Rheumatism (EULAR) response criteria were used in the assessment.

After 28 weeks of etanercept therapy, Wolbink's team found that the mean improvement in DAS28 score was significantly higher in anti-TNF-naive patients than in switchers $(2.1 \pm 1.3$ versus $1.61 \pm 1.4 ; P=0.015)$. This difference was mainly attributable to switchers without anti-drug antibodies, who had significantly less change in DAS28 score than anti-TNF-naive patients $(2.1 \pm 1.3$ versus $1.2 \pm 1.3 ; P=0.001)$. By contrast, no significant difference was observed between anti-TNF-naive patients and the subgroup of switchers with antibodies to a previous anti-TNF agent $(2.1 \pm 1.3$ versus $2.0 \pm 1.3 ; P=0.743)$. Furthermore, according to EULAR response criteria, a greater percentage of switchers without anti-drug antibodies did not respond to etanercept compared with those with anti-drug antibodies (33\% versus $9 \%$; $P=0.014)$.

The researchers concluded that the presence or absence of anti-drug antibodies from treatment with a previous anti-TNF agent can influence the success of treatment with a subsequent anti-TNF drug. "There is a need for the general availability of simple and standardized assays for drug levels and immunogenicity testing in patients treated with immunogenic therapeutic antibodies," remarks Wolbink, who goes on to say that "these assays will enable clinicians to discriminate between different types of nonresponders."

Rowan Higgs

Original article Jamnitski, A. et al. The presence or absence of antibodies to infliximab or adalimumab determines the outcome of switching to etanercept. Ann. Rheum. Dis. doi:10.1136/ard.2010.135111 